\title{
Use of effective-capacitance variation as a measure of state-of-health in a series-connected automotive battery pack
}

\author{
Peter Leijen AECS Ltd. 897 Valley Road Hastings, New Zealand peter@aecs.net \\ D. Alistair Steyn-Ross School of Engineering The University of Waikato Hamilton, New Zealand \\ asr@waikato.ac.nz \\ Nihal Kularatna School of Engineering The University of Waikato Hamilton, New Zealand \\ nihalkul@waikato.ac.nz
}

\begin{abstract}
In electric and hybrid-electric vehicles, seriesconnected battery packs are commonly used. Should the stateof-health (SOH) of one or several individual cells deteriorate, the entire battery pack is affected, reducing battery pack capacity which in turn reduces the maximum distance able to be driven. In order to predict the SOH of the individual battery cells, this paper introduces the concept of effective battery capacitance. Effective capacitance is defined as the local slope of the voltage vs charge curve derived from a third-order polynomial relationship between these parameters. The location of maximum effective capacitance can be used as a means of identifying end-of-life and/or catastrophic failure of battery modules. Four different Toyota Prius battery packs were used in establishing this method in the proof of concept work. The paper presents a linear relationship between maximum effective capacitance and SOH; this relationship is confirmed with a larger dataset.
\end{abstract}

Keywords - Hybrid Electric Vehicles, Battery Management, Battery Model, Slope Analysis, Effective Capacitance

\section{NOMENCLATURE}

$\begin{array}{ll}\begin{array}{l}\text { cell } \\ \text { module }\end{array} & \begin{array}{l}\text { single NiMH cell, usually } 1.2 \mathrm{~V} \text { nominal } \\ \text { six series-connected NiMH cells, } 7.2 \mathrm{~V} \text { nomi- } \\ \text { nal } \\ \text { two series-connected NiMH modules, } 14.4 \mathrm{~V} \\ \text { block }\end{array} \\ \begin{array}{l}\text { nominal } \\ \text { charge }\end{array} & \begin{array}{l}\text { integral of current with respect to time } \\ \text { charge at } 100 \% \mathrm{SOC}\left(Q_{\max }\right)\end{array} \\ \text { SOC } & \begin{array}{l}\text { state-of-charge, the charge remaining within } \\ \text { the cell as a percentage of actual capacity } \\ \text { state-of-health, the actual capacity as a percent- } \\ \text { age of rated capacity }\end{array} \\ \text { SOH } & \end{array}$

\section{INTRODUCTION}

Increasing interest in both hybrid electric vehicles (HEV) and electric vehicles (EV) means that accurate and appropriate measures of battery health are essential, particularly as these vehicles age. Many recent reviews of state-of-art battery health measurement techniques and research results have been published [1]-[3]. Barré et al [1] describes how battery ageing manifests as capacity fade and resistive film growth. Capacity fade is the loss of active material within the battery; resistive film growth is the accumulation of resistive contaminants on the battery electrodes arising from unwanted side reactions.
Capacity fade reduces the energy storage capability of the battery whereas resistive film growth reduces the maximum power available at the battery terminal due to increased internal resistance of a cell. These two performance-limiting phenomena are affected by different environmental conditions and usage patterns, making ageing assessment a difficult task [1]. Rezvanizaniani et al [2] assert that a battery's internal electrochemical processes are both nonlinear and time-varying, making them nearly impossible to observe for the purpose of predicting individual battery block failures in series-connected automotive battery packs such as the example of Toyota Prius. This paper aims to simplify the nonlinear aspects into a simple effective capacitance $\left(C_{\text {eff }}\right)$ measurement explained in section III. The following paragraphs cover state-of-the-art battery analysis techniques and provide a justification for the effective capacitance measure.

Waag et al [3] provides an extensive review of methods used to monitor batteries in EV and HEV applications; although that review covers lithium-ion batteries, the information provided is still relevant to nickel-metal-hydride (NiMH) batteries, the focus of this paper. The three review papers [1]-[3], surveying 669 references, agree that electrochemical methods, circuit models and statistical approaches are the main battery assessment practices employed in laboratory applications. Statistical methods do not work well in the case of prognostics in automotive battery packs because prior knowledge (current profile, temperature exposure, etc) of battery usage is usually unavailable. Some researchers [4]-[7] start with a fresh cell to be aged throughout the experiment. This is a valid approach to determine the nonlinear age-dependent responses of the battery using either electrochemical or circuit modelling methods. However, relationships between electrochemical behaviour or circuit components and age were not available for the present work making it difficult to map electrochemical or circuit model results to an accurate battery state-of-health. The Toyota Prius (the focus of this study) for example, does not store this data within the battery management system (BMS). Other manufacturers or vehicles sold in different countries may make battery degradation statistics available to the end user or diagnostic technician. However, little or no literature is available regarding how these statistics are collected or how much diagnostic information is retained. 


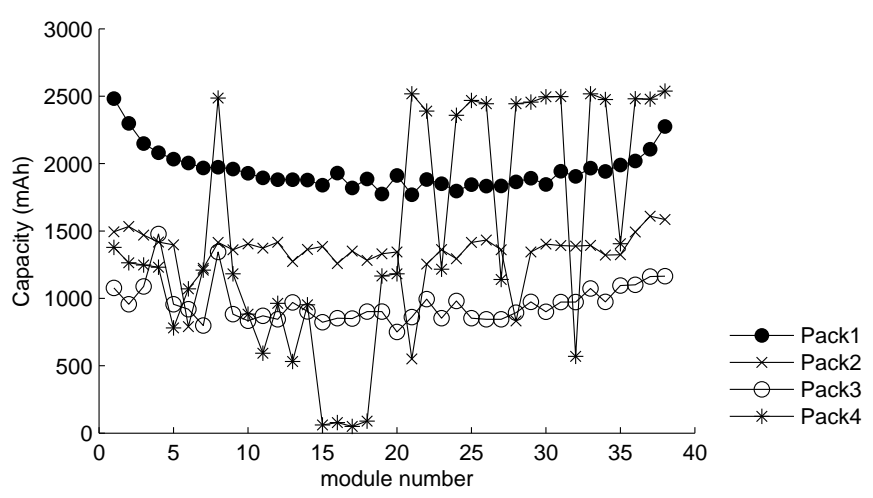

Fig. 1: Capacity distribution of aged battery packs from four different Prius vehicles. Pack 1 shows "bath-tub" (concave) capacity distribution due to accelerated ageing of battery modules in the centre of the pack (where the temperature is higher). Pack 2 shows a similar concave relationship as Pack 1, however some modules are failing through other mechanisms. Pack 3, which is more degraded than Pack 1 , also shows the concave capacity distribution. Pack 4 shows a faulty pack in which some modules retain good health whereas others have failed completely with less than $200 \mathrm{mAh}$ capacity. Capacity was measured by coulomb counting, see text for details.

Figure 1 shows the measured charge capacities for four Prius HEV battery packs, each exhibiting distinct state-of-health profiles. The capacity graphs were obtained by integrating the current versus time curve to determine, for each of the 38 battery modules per pack, the total charge $Q=\int I d t$ (coulombs) transferred during transition from a fully-charged to fullydischarged module. This measurement is often referred to as "coulomb counting". This coulomb count was then mapped to an effective module capacity in mAh via the conversion $Q_{\mathrm{mAh}}=Q \times \frac{1000}{3600}$. Each module was discharged using 4-A pulses of $35 \mathrm{~s}$ duration every $180 \mathrm{~s}$, thus allowing time for the module to settle between pulses. (Note that the nominal battery-pack capacities are unknown to field diagnostics staff.)

Packs 1, 2 and 3 have aged in a similar way: the modules near the ends of the packs have higher capacities than the modules in the middle, but are otherwise uniform. The "bathtub" curve appears to arise from the temperature differences that develop across the pack during normal operation. As argued in [8], the effective battery-pack capacity is limited by the lowest capacity module (or cell) in the series arrangement. Reference [9] details how simple measurements using commercially available diagnostic tools can give an indication of module capacity-distribution across a battery pack.

The Prius HEV battery pack consists of 38 (or 28, depending on Prius model) series-connected battery modules; each module contains six series-connected NiMH cells. As shown in Fig. 2, the battery management system (BMS) is configured to monitor the terminal voltage of pairs of modules, known as blocks, thus, unless the pack is dismantled (as was done to obtain the data for Fig. 1), the charge status of the 38 individual modules is not normally accessible for the diagnostic teams working in repair centers. Instead, the BMS can only determine end-of-charge and end-of-discharge for each of the 19 module-pairs.

The BMS assesses a pack as being "fully charged" when any

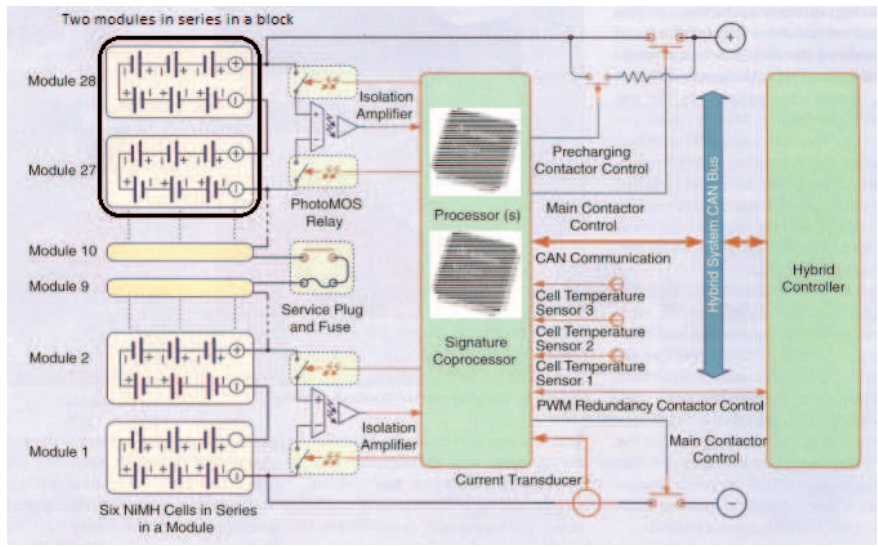

Fig. 2: A typical battery management system for HEV-2009 Toyota Prius showing the 6 series-connected cells in a battery module and the two series connected modules in a battery block [10]

one of its 19 blocks, say block-A, has a terminal voltage that has reached the nominal voltage corresponding to $100 \%$ SOC for the given cell chemistry; this means that the remaining 18 blocks will be sitting at less than full charge. Similarly, the pack is "fully discharged", when any one of the blocks, say block-B, has decayed to $0 \%$ SOC, leaving the other 18 blocks incompletely discharged. The time-integral of discharge current between the instant when block-A is fully charged (100\% SOC) to when block-B is fully discharged (0\% SOC) gives the overall pack capacity.

If one cell in a series-connected pack is of significantly lower state-of-health, the charge transfer required to traverse from BMS detection of "full charge" to "complete discharge" will be substantially reduced, thus lowering the accessible capacity of the entire pack. Zheng et al [11] detail how capacity variation in series-connected cells can affect overall pack performance. While various advanced techniques such as the hardware in the loop (HIL) simulation could be applied in cases where BMS designers are ready to apply them bearing the added design cost in mind, in an off-line diagnostic exercise such as this case, such techniques are not economically and practically viable.

In this paper we describe a direct and simple method for assessing battery state-of-health from the inverse-slope of the voltage-versus-charge (i.e., integrated current) discharging curves. This inverse-slope defines an effective battery capacitance $C_{\text {eff }}=(d V / d Q)^{-1}$. This is for a real-world diagnostic exercise in the field service and repairs of automotive battery packs to pin-point the failing individual battery blocks. We show that a healthy battery-pack has a relatively flat $V$ vs- $Q$ characteristic and large effective capacitance, whereas an unhealthy pack exhibits a terminal voltage that is much more sensitive to charge transfer, so has diminished $C_{\text {eff. }}$ This practically and industrially useful new method is suitable for use as a field diagnostic since it does not require access to specialist laboratory equipment or to high-order matrix-based numerics typically needed for parameter extraction via Kalman and extended Kalman filter modeling. 


\section{Model-Based Approach to State-Of-Health MeAsurement}

The most common battery model described in the literature is shown in Figure 3(a) [12]-[27]. In [19] this model is referred to as the diffusion-polarisation (DP) model where, $V_{\mathrm{o}}$ is the open-circuit terminal voltage, $R_{\mathrm{T}}$ is the terminal resistance, $R_{\mathrm{D}}, C_{\mathrm{D}}$ and $R_{\mathrm{P}}, C_{\mathrm{P}}$ are the diffusion and polarisation resistances and capacitances respectively. This model is used to describe the small-signal behaviour of a battery about some DC bias point, taken in this case as the $I=0$ steady-state terminal voltage i.e., $V_{o}$.

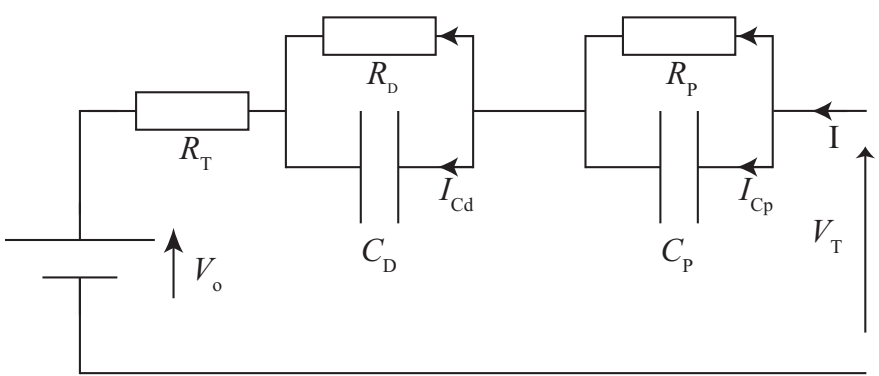

(a)

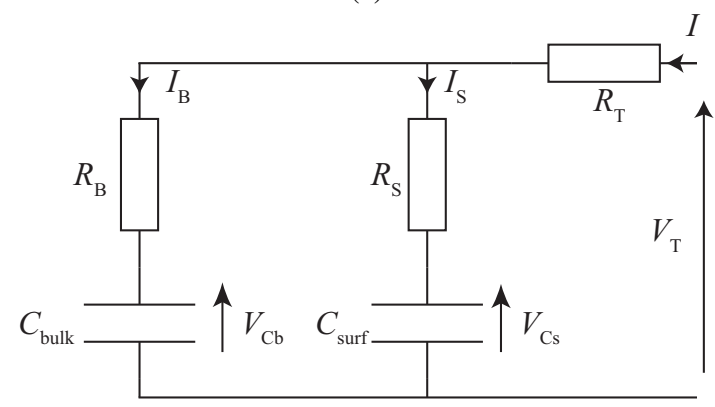

(b)

Fig. 3: Two commonly-used battery models. (a) Thévenin or diffusion-polarisation model describes the small-signal voltage recovery after a current pulse. Bulk-surface model (b) describes the bulk behaviour of the battery due to capacitor energy storage by linking charge to terminal voltage [28], [29].

Many of the aforementioned research papers establish a link between the internal parameters of the battery model shown in Figure 3(a) and state-of-charge (SOC). As battery SOC changes, so does the open-circuit voltage a characteristic which the DP model is unable to reconstruct accurately. This behaviour can be approximately modelled by replacing the fixed voltage source $\left(V_{o}\right)$ in Figure 3(a) with a bulk storage capacitor. Hence, the approximate model in Figure 3(b) is more suitable for practically modelling large-signal battery behaviour where, $R_{\mathrm{B}}$ and $C_{\text {bulk }}$ are the bulk resistance and capacitance which together form a low frequency low-pass filter thus describing the slow responses (charging and discharging process) of the battery. The surface $R C$ ( $R_{\mathrm{S}}$ and $C_{\text {surf }}$ ) pair reconstructs the high frequency behaviour of the battery i.e., terminal voltage fluctuations due to sudden changes in terminal current. These two battery models can be related through a star-delta transformation [28]. Figure 3(b) is adapted from Bhangu et al. [29] who use an extended Kalman filtering (EKF) approach to quantify the bulk-storage capacitance, and to assess battery state-of-health. However, for the EKF to work correctly, an accurate estimate of other battery parameters (surface capacitance and series resistances) is also required. Estimated parameter values were used in [29] and a broad assumption is taken that the other battery parameters remain constant as the battery ages.

Modelling the battery using large capacitors, as in Figure 3 (b), relates the terminal voltage $\left(V_{\mathrm{T}}\right)$ to state-of-charge approximating a more realistic battery. However, when we look closely at the charge and discharge curves (Fig. 4) we see that the relationship between state-of-charge and open-circuit voltage does not follow the linear trend expected of a fixed capacitor. It is observed that $C_{\text {bulk }} \gg C_{\text {surf }}$ hence the effects of the surface capacitance on battery storage capability can be considered negligible. The storage capacitor must instead be modelled by some nonlinear function with multiple inputs,

$$
C_{\text {bulk }}=f(\text { charge }, V, \text { SOH, temp }, \mathrm{X}),
$$

where " $X$ " represents the remaining battery parameters such as charge-rate or hysteresis effects [24].

The battery models presented in Figure 3 are appropriate for single-cell research where only the voltage responses of two (positive and negative) electrodes are considered, but cannot directly extend to multi-cell batteries [1]. Practical automotive batteries rarely have terminals exposed to allow individual measurements at cell level. Lithium technologies are an exception due to the dangers of single cells reaching run-away temperatures resulting from over-charge or excessive discharge. As indicated before, Toyota Prius battery modules consist of six series-connected NiMH cells; two seriesconnected module constitute a block. As a result equation (1) becomes increasingly complex. If there is no difference between cells then we could consider the module as a single cell, however in reality individual cells are never identical [11]. The total bulk capacitance of the battery block now becomes a function of the 12 individual bulk capacitances,

$$
C_{\text {bulk }}^{\text {tot }}=f\left(\left.C_{\text {bulk }}\right|_{1},\left.C_{\text {bulk }}\right|_{2}, \ldots,\left.C_{\text {bulk }}\right|_{12}\right),
$$

which in turn becomes a function of the 12 individual battery parameters,

$$
C_{\text {bulk }}^{\text {tot }}=f\left(\left.\operatorname{charge}\right|_{i},\left.V\right|_{i},\left.\mathrm{SOH}\right|_{i}, \text { temp }\left.\right|_{i},\left.\mathrm{X}\right|_{i}\right)
$$

where the $\left.\right|_{i}$ notation symbolises the parameter evaluated at cell $i$ where $i=1,2, \ldots, 12$ for the 12 series-connected cells. Building a model to estimate all of these individual parameters, given only battery terminal current and voltage, and ambient temperature, is possible using advanced error minimisation techniques such as extended Kalman filtering. However this is not practical because of the computational requirements to process at least order 12 matrices. Hence a new grouped parameter, the effective capacitance, is proposed as a measure of battery state-of-health. This new measure is useful in practical systems such as the Prius. 


\section{EfFective Capacitance Measure}

Little work has been done on the development of suitable off-line techniques for determining state-of-health of a seriesconnected multi-cell battery. Most battery models presented in the literature contain some form of current-dependant voltage source such as, in its simplest form, a capacitor (Figure 3(b)). Many models such as that presented in [29] relate this storage element to state-of-health. However due to the nonlinear interaction between the multiple battery-model components of Eq. (1), it is extremely challenging to parameterise its capacitance. A determination of the "effective capacitance" is presented here as a practically reliable indicator of stateof-health of a battery block (in our case 12 series-connected $\mathrm{NiMH}$ cells), used in applications such as the Toyota Prius battery pack.

We define effective capacitance as the local slope of the charge-vs-voltage curve,

$$
C_{\mathrm{eff}}=\frac{d Q}{d V}
$$

which is the small-signal equivalent of the bulk capacitance definition, $C=Q / V$. Here, $C_{\text {eff }}$ takes into account any inherent nonlinearities because it is calculated on a point-bypoint basis along the discharge curve. Figure 4 shows how $C_{\text {eff }}$ deviates from the linear relationship expected between state-of-charge and voltage for an ordinary capacitor.

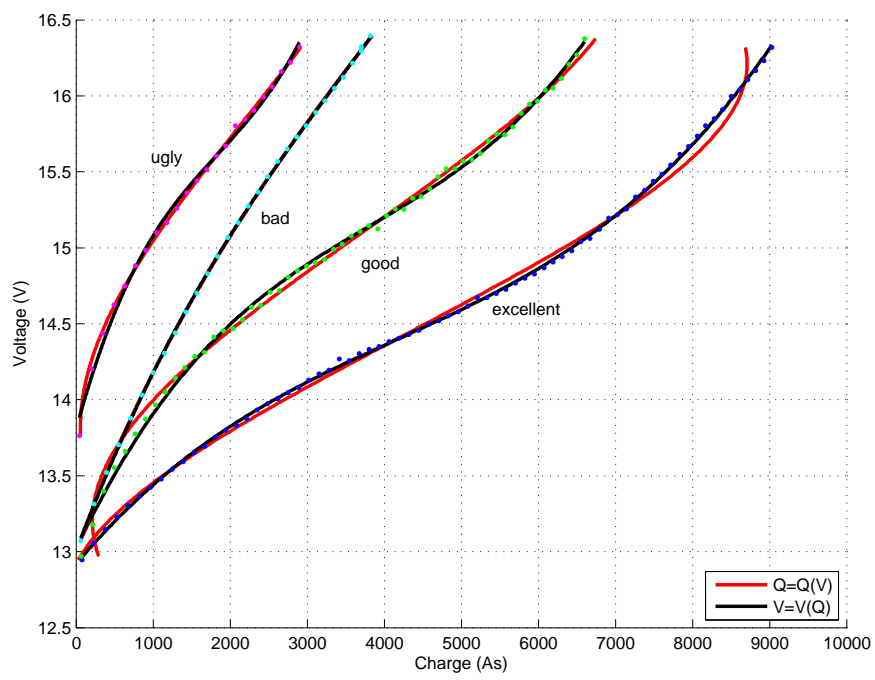

Fig. 4: Discharge curves comparing third-order polynomial fits $Q=$ $Q(V)$ and $V=V(Q)$ for four battery blocks (series-connected pairs of modules) whose states-of-health are, from visual inspection, grouped into four categories. The basis for this grouping is on length and shape of the discharge curve. Note: The colour scheme identifying the family of $(Q, V)$ data points is retained in Fig 5.

This effective-capacitance method was developed after analysing data obtained from a supercapacitor-based off-line battery diagnostic tool [30] implemented in the first stage of this project. The diagnostic system connects to the sensing wires on the Toyota Prius battery pack to enable voltage and current measurements at each battery-block terminal.

If we knew the true functional form for $Q(V)$, batterycharge as a function of battery-voltage, then we could use simple calculus to compute the capacitance from the gradient of the $Q$-vs- $V$ curve. But we do not know $Q(V)$; instead we have a sequence of point values for $(V, Q)$ along the discharge path, mapping the real nature of the grouped-capacitance variations. These point values are inevitably influenced by measurement noise, so a simple ratio of first-differences will produce very noisy gradient estimates. Therefore we need to apply a smoothing technique to the measurements to increase the accuracy of the gradient estimates. Inspection of the curves, Figure 4, suggests that, over the range of these measurements, the voltage $V$ could be approximately expressed as a cubic polynomial of charge $Q$,

$$
V(Q)=a_{3} Q^{3}+a_{2} Q^{2}+a_{1} Q+a_{0},
$$

or alternatively charge can be approximately expressed as a cubic polynomial of voltage,

$$
Q(V)=b_{3} V^{3}+b_{2} V^{2}+b_{1} V+b_{0}
$$

It can be seen in Figure 4 that both equations (5) and (6) match the measured data reasonably well. Using calculus we can now determine the effective capacitance. From equation (6),

$$
C_{\text {eff }}=\frac{d Q}{d V}=3 b_{3} V^{2}+2 b_{2} V+b_{1}
$$

which is a quadratic relationship between voltage and $C_{\text {eff }}$, implying that $C_{\text {eff }}$ can approach $+\infty$ or $-\infty$ when $V \rightarrow \infty$, depending on the sign of the $b_{3}$ coefficient. Such unbounded behaviour for $C_{\text {eff }}$ is clearly undesirable.

Fortunately the $V(Q)$ fitting produces a bounded and well-

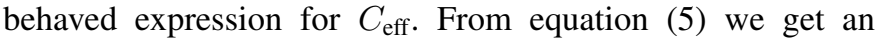
inverse-quadratic function,

$$
C_{\mathrm{eff}}=\left(\frac{d V}{d Q}\right)^{-1}=\frac{1}{3 a_{3} Q^{2}+2 a_{2} Q+a_{1}},
$$
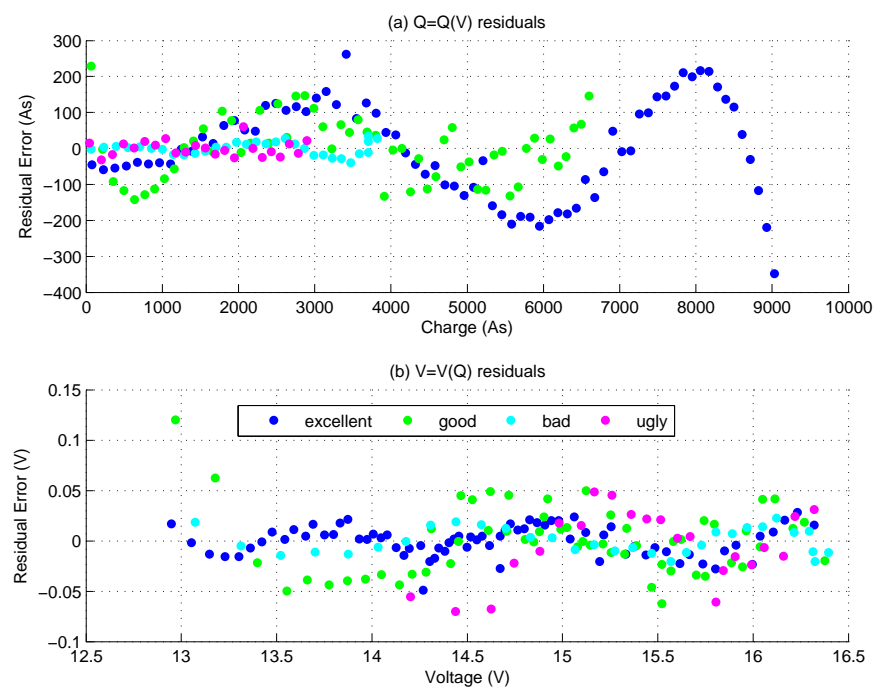

Fig. 5: Residual error between measurements and (a) $Q=Q(V)$ cubic fit, and (b) $V=V(Q)$ cubic fit for the four battery blocks shown in Figure 4 confirming that $V=V(Q)$ is the better approximation for this dataset. 
which is a bell-shaped curve with an asymptote at $C_{\text {eff }}=0$. If we set $Q=0$ then $C_{\text {eff }}=1 / a_{1}$, which is positive for all discharge curves; we might interpret this as the component of the capacitance of the cell that is not related to any chemical changes. For example, $\left.C_{\text {eff }}\right|_{Q=0}$ could be an indication of plate area, separation distance, and electrolyte permittivity, where smaller $a_{1}$ values indicate a higher capacity battery i.e. larger plate area, smaller separation distance or better electrolyte permittivity. Additionally, the charge at which the $C_{\text {eff }}$-vs$Q$ curve reaches its maximum $\left(\left.Q\right|_{C_{\text {eff }}^{\max }}\right)$ can be calculated by locating the turning-point,

$$
\frac{d C_{\mathrm{eff}}}{d Q}=-\frac{6 a_{3} Q+2 a_{2}}{\left(3 a_{3} Q^{2}+2 a_{2} Q+a_{1}\right)^{2}}=0,
$$

so,

$$
\left.Q\right|_{C_{\mathrm{eff}}^{\max }}=\frac{-a_{2}}{3 a_{3}}
$$

therefore,

$$
C_{\mathrm{eff}}^{\max }=\frac{3 a_{3}}{3 a_{1} a_{3}-a_{2}^{2}}
$$

where a larger $C_{\mathrm{eff}}^{\max }$ indicates a healthier battery i.e., better energy storage capability. From equation (5) we can see that $a_{0}$ is the terminal voltage of the cell when there is no charge.
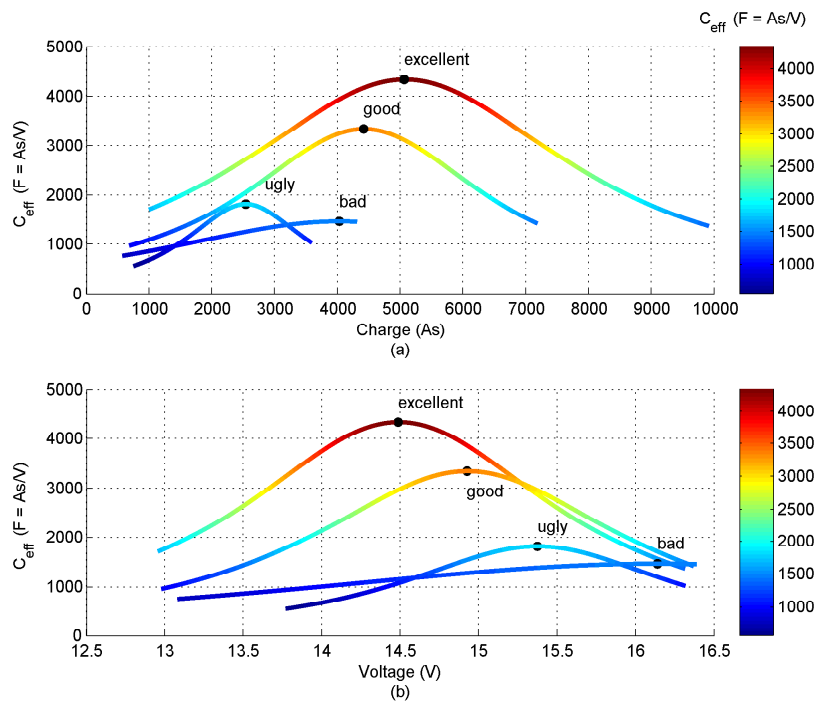

Fig. 6: Effective capacitance calculated using $C_{\text {eff }}=(d V / d Q)^{-1}$ the cubic polynomial fit $V(Q)=a_{0}+a_{1} Q+a_{2} Q^{2}+a_{3} Q^{3}$ plotted against (a) charge and (b) voltage for the four battery blocks shown in Figure 4. The dots mark the location of maximum effective capacitance, $C_{\text {eff }}^{\max }$, and the colour scale indicates the magnitude of $C_{\text {eff. The same }}$ colour scheme is used in Figure 8.

In order to confirm this measurement technique, we have tentatively identified four battery modules as "excellent", "good", "bad" and "ugly" after referring to their apparent stateof-health based on inspection of their respective discharge curves plotted in Figure 4. Figure 4 shows that "ugly" has a higher value for $a_{0}$, but careful inspection of Figure 8(b) shows that batteries that suffered catastrophic failures, end-oflife failures and ordinary ageing present with similar values for $a_{0}$, hence there is no direct relationship between $a_{0}$ and battery health.

Graphs of the residual error for the two fits of Figure 4 are shown in Figure 5. The maximum residual error, expressed as a percentage, for $Q=Q(V)$ fit is $4.4 \%$ and for $V=V(Q)$ fit is $0.9 \%$ confirming that $V=V(Q)$ gives a more accurate description of the voltage-discharge characteristics for these battery blocks.

Effective capacitance trends calculated from the local gradients of the cubic $V=V(Q)$ polynomial fits of Figure 4 are shown in Figure 6 plotted against charge and voltage. The maximum effective capacitances are marked with a dot. $\left[C_{\mathrm{eff}}^{\max }\right.$ was also calculated using the $Q=Q(V)$ cubic fit (shown in Fig. 4), and we found a maximum difference between the values for $C_{\mathrm{eff}}^{\max }$ (slope at the point of inflection) of 17\%, (not shown).]

Maximum effective capacitance (highlighted by the dots on Fig 6) occurs where the battery voltage response is most linear around the point of inflection, highlighting the nonlinear behaviour of the battery-model bulk-capacitance $\left(C_{\text {bulk }}\right)$ shown in Figure 3(b). This changing behaviour of the series battery block is a result of the complex electrochemical processes. A NiMH battery is fully charged when all chemical species have oxidised from $\mathrm{Ni}$ (II) to $\mathrm{Ni}$ (III), the electrolyte and the two electrodes now effectively forming a capacitor (charged plates separated by a distance). For constant-current discharge, the voltage-vs-charge curve should be linear if the cell behaves as a perfectly linear capacitor. However, this theoretical capacitor, formed by the electrodes and electrolyte, is charged by the electrochemical redox reactions occurring within the electrodes with ion diffusion through the electrolyte [31]. The chemical reactions effectively charge the theoretical capacitor and the voltage deviates from the expected linear relationship. This phenomenon highlights the electrochemical aspects of the battery and indicates that modelling the battery as a fixed capacitor is an approximation at best. We hypothesise that the location of $C_{\mathrm{eff}}^{\max }$ on the voltage-charge plane, and the value of $C_{\text {eff }}^{\max }$ itself, are primary indicators for battery state-of-health. We now test this hypothesis on 40 sets of data.

\section{RESUlTS AND Discussion}

The data in the following figures were obtained from a variety of Toyota Prius battery packs, some from vehicles that remain in service, and others from battery packs which have failed in the field. All measurements were taken at an ambient temperature of $15^{\circ} \mathrm{C}$, discharging a constant $4 \mathrm{~A}$ for 35 seconds every 180 seconds, giving the battery $145 \mathrm{~s}$ to rest and recover before recording the open-circuit voltage.

A measurement of capacity $\left(Q_{\max }\right)$ can be obtained by integrating the current between the end-of-charge and endof-discharge points,

$$
Q_{\max }=\int_{t_{0}}^{t_{1}} I(t) d t
$$

where, $t_{1}-t_{0}$ is the time taken to discharge the module from end-of-charge to end-of-discharge and $I$ is the time-varying current through the module. State-of-health is defined as, 


$$
\mathrm{SOH}=\frac{Q_{\max }}{Q_{\text {rated }}} \cdot 100 \%,
$$

but for these battery blocks $Q_{\text {rated }}$ is not readily available from battery manufacturers. So, for this battery type, $\mathrm{SOH}$ cannot be expressed as a percentage. An attempt was made at finding a relationship between the fit coefficients $\left(a_{0}, a_{1}, a_{2}\right.$ and $\left.a_{3}\right)$ and $Q_{\max }$; however no clear relationship was found. Hence a relationship between capacity and $C_{\mathrm{eff}}^{\max }$ is suggested.

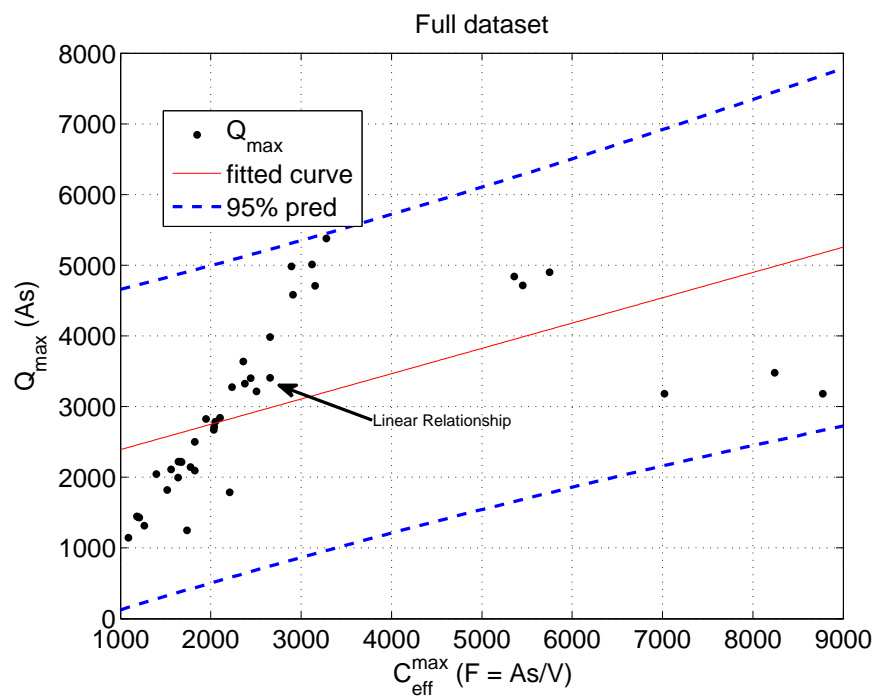

(a)

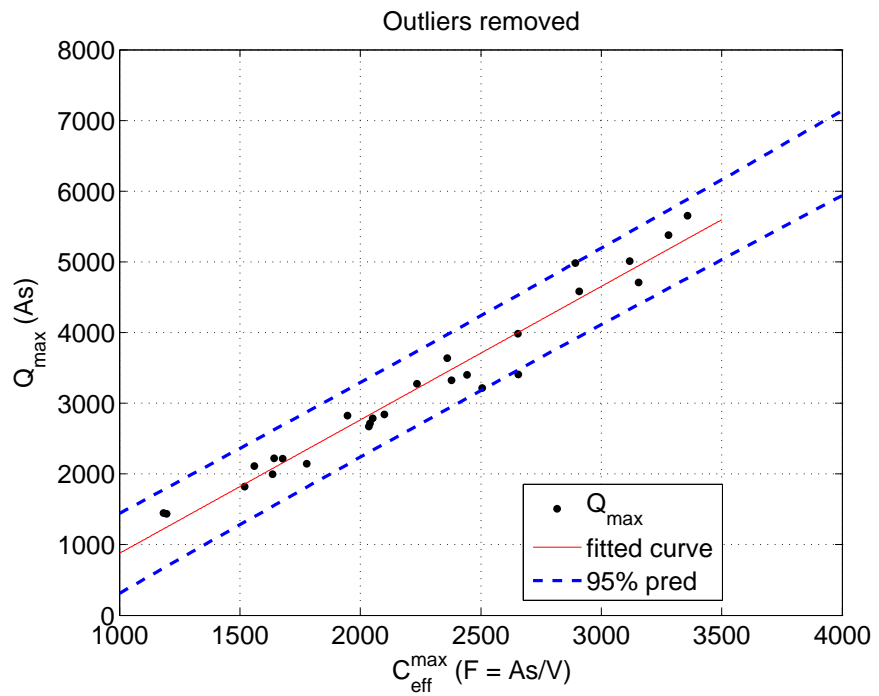

(b)

Fig. 7: Capacity, fitted curve $(m x+c)$ and $95 \%$ prediction interval plotted against local maximum $C_{\text {eff }}$ for (a) complete dataset including outliers and (b) with outliers removed. For (b), the fit parameters are $m=1.891 \mathrm{AsF}^{-1}$ and $c=-1025$ As.

Figure 7(a) shows capacity plotted against $C_{\text {eff }}^{\max }$ for the full dataset. While a linear relationship is clearly visible, it is skewed by outliers. The outlying data-points represent a number of possible failures such as short-circuited cells, reversed electrodes or end-of-life failures. Considering that the measurements are of a battery block where the individual cell terminals are not accessible we can only speculate as to which type of failure has occurred. In Figure 8 we look at the location of $C_{\mathrm{eff}}^{\max }$ on the charge-voltage plane to distinguish between catastrophic failures and end-of-life failures. Figure 7(b) removes the outliers to reveal the underlying linear relationship between capacity $Q_{\max }$ and $C_{\mathrm{eff}}^{\max }$,

$$
Q_{\max }=1.891 \cdot C_{\mathrm{eff}}^{\max }-1025
$$

Although researchers commonly describe capacity-fade ageing effects seen in battery model parameters as an exponential decay over time [7] such relationship was not suitable for this dataset as time-series ageing data are not available. If capacity reduces to zero, an effective capacitance $\left(1 / a_{1}\right)$ of $\approx 550 \mathrm{~F}$ remains; this residual $550 \mathrm{~F}$ can be pictured as the capacitance of the battery when no active species are present, so no ion transfer through the electrolyte is possible [32].

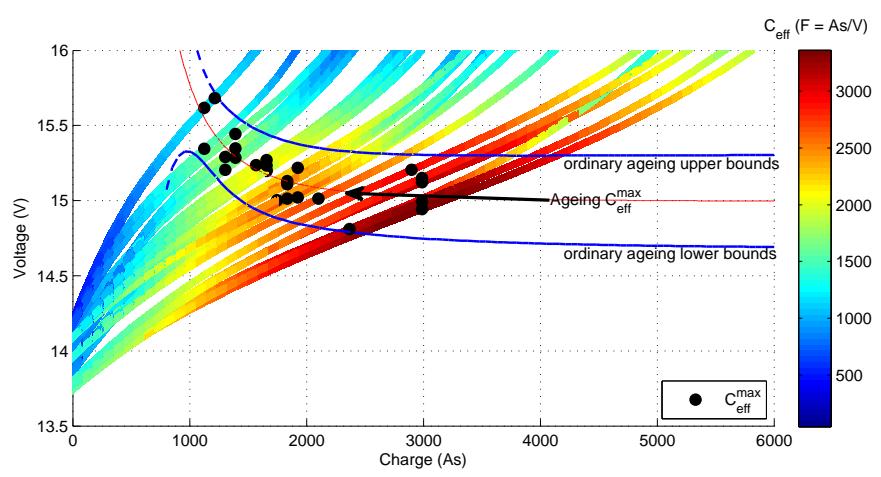

(a)

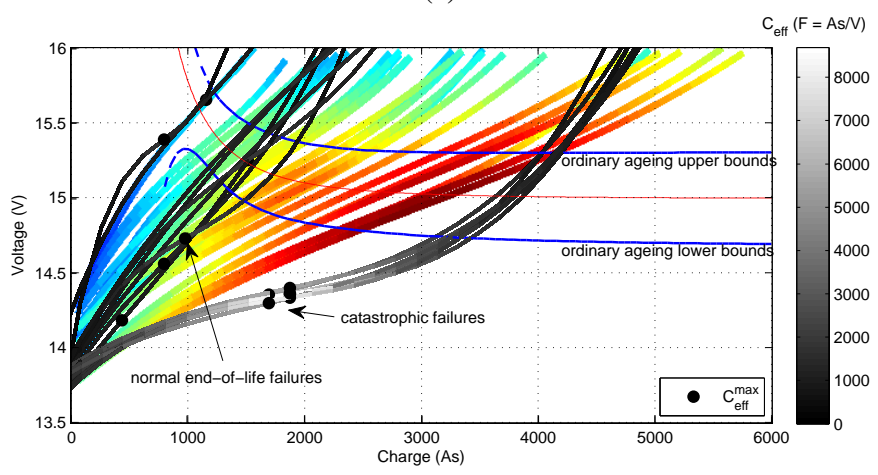

(b)

Fig. 8: Effective capacitance, indicated by the colour bars (as defined in Figure 6), plotted along the battery block discharge curve for (a) ordinary ageing dataset showing relationship between $C_{\mathrm{eff}}^{\max }$ (black dots), charge and voltage and $95 \%$ prediction interval i.e. the ordinary ageing locus and (b) with outliers (catastrophic and end-of-life failures) included. These plots highlight the unpredictable failure modes when battery-health reduces (end-of-life) and "catastrophic" battery failure such as short-circuited cells where $C_{\text {eff }}^{\max }$ falls outside ordinary ageing bounds.

The relationships between maximum effective capacitance $\left(C_{\mathrm{eff}}^{\max }\right)$ and the $\left(Q_{x}, V_{x}\right)$ coordinate at which the point of inflection occurs shown in Figure 8, with the outliers removed are,

$$
Q_{x}=1.32 \times 10^{-5} \cdot\left(C_{\mathrm{eff}}^{\max }\right)^{2.33}+934
$$


More significant however is the relationship between voltage and $C_{\text {eff }}$,

$$
V_{x}=1.1 \times 10^{4} \cdot\left(C_{\text {eff }}^{\max }\right)^{-1.34}+14.8,
$$

indicating that as battery health increases, corresponding to increasing $C_{\text {eff }}^{\max }$ in Figure $7(\mathrm{~b})$, the inflection voltage $V_{x}$ approaches $14.8 \mathrm{~V}$. For a block of 12 series-connected NiMH cells, $14.8 \mathrm{~V}$ corresponds to a nominal voltage of between 1.2 and $1.3 \mathrm{~V}$ per cell. This suggests that the cubic-fitting approach may be transferrable to different battery chemistries after appropriate adjustment to the constants in equations (15) and (16). We argue that equations (15) and (16) describe the movement of $C_{\mathrm{eff}}^{\max }$ through the voltage-charge plane as a result of ordinary battery ageing which manifests as a gradual fading of capacity. This relationship is plotted on Figure 8(a) where, only the battery blocks that fit the linear relationship from Fig. 7(b) are plotted.

Figure 8(b) superimposes the dataset outliers from Fig. 7(a). Here we can identify two clear regions: battery end-of-life and catastrophic failures. The end-of-life region is identified by battery blocks whose $C_{\text {eff }}^{\max }$ values have reduced below $\sim$ $1500 \mathrm{~F}$. The scatter in $C_{\mathrm{eff}}^{\max }$ values highlights the unpredictable behaviour for these failing battery blocks. Batteries with peak effective capacitance that fall in the end-of-life region, but also within the ordinary ageing bounds, may still be suitable for reuse, however the battery-pack may fail within a short timeframe.

The catastrophic failure region shows blocks exhibiting either short-circuited cells or electrode reversal and are identified by a high values for $C_{\text {eff }}^{\max }(\geq 4000 \mathrm{~F}$ ) lying outside of the ordinary ageing region. If one of the 12 series-connected cells is short-circuited it is still possible to charge the battery block to $16 \mathrm{~V}$ however this results in 11 severely over-charged cells. When the block is subsequently discharged the rate-of-change in voltage with respect to charge is higher as the over-charge is removed from the electrodes. The voltage at which $C_{\mathrm{eff}}^{\max }$ occurs is now lower, outside of ordinary ageing bounds, as only 11 cells contribute to the overall storage capability of the block. Electrode reversal also shows a similar accelerated drop in voltage when the battery block is discharged [33].

It must also be noted that the numerical value of $C_{\text {eff }}^{\max }$ is much higher for the catastrophic failures than ordinary cells: $8000 \mathrm{~F}$ as opposed to $3000 \mathrm{~F}$ as shown in Figure 8. This is a side effect of the $C_{\text {eff }}$ calculation, the rate-of-change of slope is much higher at the turning point for the catastrophic failure due to the steep initial voltage drop. What is of importance in this case is that $C_{\mathrm{eff}}^{\max }$ lies outside of the ordinary ageing region.

\section{CONCLUSION}

In this project authors have utilized the battery pack failure data collected in field-repair work, which practically indicated the failing blocks in series connected automotive battery packs (with Toyota Prius as the specific example) to verify the validity of a new parameter-estimation method useful for pin-pointing the failing battery blocks. By inserting outof-specification battery blocks removed in the field repair exercises, authors were able to verify the validity of this new off-line prognostic procedure to carry out an estimation of an approximate effective capacitance of a combination of Ni-MH cells.

Maximum effective-capacitance calculated from a thirdorder polynomial fit to $(Q, V)$ data-points and its position on the voltage-charge plane accurately distinguishes catastrophic and end-of-life battery failure from ordinary battery ageing processes causing capacity fade effects. The coefficients of the third-order fit $V=V(Q)$ can be used as design parameters to optimise battery pack performance. However, no clear relationship exists between the fit coefficients and state-ofhealth. Rather, the suggested method shows ordinary ageing bounds within which $C_{\text {eff }}^{\max }$ must lie, with $C_{\text {eff }}^{\max }$ having a positive linear relationship with $\mathrm{SOH}$ capacity. These bounds are calculated through a power-law relationship between $C_{\mathrm{eff}}^{\mathrm{max}}$, $V_{x}$ and $Q_{x}$.

\section{ACKNOWLEDGMENT}

Authors would like to thank Toyota New Zealand, Hyde Automotive and Mudgeway for providing the battery packs used during the experiments. We would also like to thank AECS Ltd for providing valuable assistance with the interpretation of the large amount of test data and the use of their test equipment. We acknowledge the New Zealand Ministry of Business Innovation and Enterprise for funding support.

\section{REFERENCES}

[1] A. Barré, B. Deguilhem, S. Grolleau, M. Gérard, F. Suard, and D. Riu, "A review on lithium-ion battery ageing mechanisms and estimations for automotive applications," Journal of Power Sources, vol. 241, pp. $680-689,2013$.

[2] S. M. Rezvanizaniani, Z. Liu, Y. Chen, and J. Lee, "Review and recent advances in battery health monitoring and prognostics technologies for electric vehicle (EV) safety and mobility," Journal of Power Sources, vol. 256, pp. 110-124, 2014.

[3] W. Waag, C. Fleischer, and D. U. Sauer, "Critical review of the methods for monitoring of lithium-ion batteries in electric and hybrid vehicles," Journal of Power Sources, vol. 258, pp. 321-339, 2014.

[4] M. V. Micea, L. Ungurean, G. N. Carstoiu, and V. Groza, "Online stateof-health assessment for battery management systems," IEEE Transactions on Instrumentation and Measurement, vol. 60, no. 6, pp. 1997 2006, June 2011.

[5] H.-T. Lin, T.-J. Liang, and S.-M. Chen, "Estimation of battery state of health using probabilistic neural network," IEEE Transactions on Industrial Informatics, vol. 9, no. 2, pp. 679 - 685, May 2013.

[6] J. Kin and B. H. Cho, "State-of-charge estimation and state-of-health prediction of a Li-ion degraded battery based on an EKF combined with a per-unit system," IEEE Transactions on Vehicular Technology, vol. 60, no. 9, pp. 4249 - 4260, November 2011.

[7] P. Ramadass, B. Haran, R. White, and B. N. Popov, "Mathematical modeling of the capacity fade of Li-ion cells," Journal of Power Sources, vol. 123 , no. 2, pp. $230-240,2003$

[8] P. Leijen and J. Scott, "Failure analysis of some Toyota Prius battery packs and potential for recovery," SAE Technical Paper 2013-01-2561, 2013.

[9] P. Leijen, "Real world battery diagnostics model based and prius case study," IEEE International Symposium on Industrial Electronics (ISIE), pp. 1-6, 2014.

[10] J. Cao and A. Emadi, "Batteries need electronics," IEEE Industrial Electronics, vol. 5, no. 1, pp. 27-35, March 2011.

[11] Y. Zheng, L. Lu, X. Han, J. Li, and M. Ouyang, "LiFePO4 battery pack capacity estimation for electric vehicles based on charging cell voltage curve transformation," Journal of Power Sources, vol. 226, pp. 33-41, 2013. 
[12] B. Schweighofer, K. M. Raab, and G. Brasseur, "Modeling of high power automotive batteries by the use of an automated test system," IEEE Transactions on Instrumentation and Measurement, vol. 52, pp. 10871091, 2003

[13] H. Li, C. Liao, and L. Wang, "Research on state-of-charge estimation of battery pack used on hybrid electric vehicle," Power and Energy Engineering Conference, pp. 1-4, March 2009.

[14] L. Serrao, Z. Chehab, Y. Guezennee, and G. Rizzoni, "An aging model of Ni-MH batteries for hybrid electric vehicles," IEEE Conference on Vehicle Power and Propulsion, 2005.

[15] L. Gao, S. Liu, and R. A. Dougal, "Dynamic lithium-ion battery model for system simulation," IEEE Transactions on Components and Packaging Technologies, vol. 25, pp. 495-505, 2002.

[16] K. Bundy, M. Karlsson, G. Lindbergh, and A. Lundqvist, "An electrochemical impedance spectroscopy method for prediction of the state of charge of a nickel-metal hydride battery at open circuit and during discharge," Journal of Power Sources, vol. 72, no. 2, pp. 118-125, 1998.

[17] A. Eddahech, O. Briat, N. Bertrand, J.-Y. Deletage, and J.-M. Vinassa, "Behavior and state-of-health monitoring of Li-ion batteries using impedance spectroscopy and recurrent neural networks," International Journal of Electrical Power \& Energy Systems, vol. 42, no. 1, pp. 487 - 494, November 2012.

[18] W. Guoliang, L. Rengui, Z. Chunbo, and C. Chan, "State of charge estimation for NiMH battery based on electromotive force method," IEEE Conference on Vehicle Power and Propulsion, pp. 1-5, September 2008.

[19] H. He, R. Xiong, H. Guo, and S. Li, "Comparison study on the battery models used for the energy management of batteries in electric vehicles," Energy Conversion and Management, vol. 64, pp. 113 - 121, 2012, iREC 2011, The International Renewable Energy Congress.

[20] F. Huet, "A review of impedance measurements for determination of the state-of-charge or state-of-health of secondary batteries," Journal of Power Sources, vol. 70, no. 1, pp. 59 - 69, January 1998.

[21] J. Remmlinger, M. Buchholz, M. Meiler, P. Bernreuter, and K. Dietmayer, "State-of-health monitoring of lithium-ion batteries in electric vehicles by on-board internal resistance estimation," Journal of Power Sources, vol. 196, no. 12, pp. 5357 - 5363, June 2011.

[22] A. J. Salkind, C. Fennie, P. Singh, T. Atwater, and D. E. Reisner, "Determination of state-of-charge and state-of-health of batteries by fuzzy logic methodology," Journal of Power Sources, vol. 80, no. 12, pp. 293 - 300, July 1999.

[23] A. Tenno, R. Tenno, and T. Suntio, "Battery impedance characterization through inspection of discharge curve and testing with short pulses," Journal of Power Sources, vol. 158, no. 2, pp. 1029 - 1033, 2006.

[24] M. Thele, O. Bohlen, D. U. Sauer, and E. Karden, "Development of a voltage-behavior model for NiMH batteries using an impedance-based modeling concept," Journal of Power Sources, vol. 175, no. 1, pp. 635643, January 2008.

[25] M. Verbrugge and E. Tate, "Adaptive state of charge algorithm for nickel metal hydride batteries including hysteresis phenomena," Journal of Power Sources, vol. 126, no. 1-2, pp. 236-249, February 2004.

[26] Y. Hu, S. Yurkovich, Y. Guezennec, and B. J. Yurkovich, "Electrothermal battery model identification for automotive applications," Journal of Power Sources, vol. 196, no. 1, pp. 449-457, 2011.

[27] T. Hu and H. Jung, "Simple algorithms for determining parameters of circuit models for charging/discharging batteries," Journal of Power Sources, vol. 233, pp. 14-22, 2013.

[28] C. R. Gould, C. M. Bingham, D. A. Stone, and P. Bentley, "New battery model and state-of-health determination through subspace parameter estimation and state-observer techniques," IEEE Transactions on Vehicular Technology, vol. 58, no. 8, pp. 3905 - 3916, October 2009.

[29] B. S. Bhangu, P. Bentley, D. A. Stone, and C. M. Bingham, "Nonlinear observers for predicting State-of-Charge and State-of-Health of lead-acid batteries for hybrid-electric vehicles," IEEE Transactions on Vehicular Technology, vol. 54, no. 3, pp. 783-794, May 2005.

[30] P. Leijen and N. Kularatna, "Developing a monitoring system for Toyota Prius battery-packs for longer term performance issues," IEEE International Symposium on Industrial Electronics (ISIE), pp. 1-6, 2013.

[31] A. Ledovskikh, E. Verbitskiy, A. Ayeb, and P. H. L. Notten, "Modelling of rechargeable NiMH batteries," Journal of Alloys and Compounds, vol. 356-357, pp. 742-745, August 2002.

[32] P. Krüsemann, A. Mank, A. Belfadhel-Ayeb, and P. Notten, "Low volume sampling device for mass spectrometry analysis of gas formation in nickel-metalhydride (NiMH) batteries," Analytica Chimica Acta, vol. 566, no. 2, pp. 238-245, 2006.

[33] D. Linden and T. B. Reddy, Handbook of Batteries, 3rd ed. McGrawHill, 1995.
Copyright (c) 2015 IEEE. Personal use of this material is permitted. However, permission to use this material for any other purposes must be obtained from the IEEE by sending a request to pubs-permissions@iee.org.

Peter Leijen is a technical

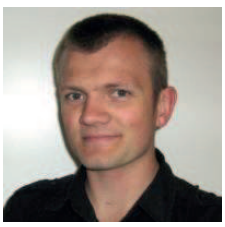
trainer/application engineer at AECS Ltd, New Zealand. His area of expertise is automotive diagnostics using both oscilloscopes and scantools. His primary focus is training automotive diagnosticians in subject areas ranging from battery technology to CAN databus systems. His secondary focus is developing new training aids namely custom vehicle simulators.

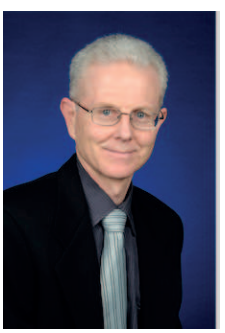

Alistair Steyn-Ross is an associate professor in physics in the School of Engineering at University of Waikato, New Zealand. His area of expertise is nonlinear modelling and computational physics techniques applied to biological systems and electronic devices. The biological research focuses on mathematical modelling of the cortex during changes of states of vigilance at onset of sleep, anaesthesia, and seizure. The electronics research analyses novel applications of supercapacitors and other nonlinear circuit elements to implement robust, energy-efficient power supplies.

Nihal Kularatna (SM98) is an Associate

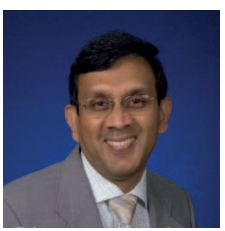

Professor in electrical engineering lecturer in the School of Engineering at the University of Waikato, New Zealand. A recipient of the New Zealand innovator of the year award for 2013, his electronic engineering career spans 42 years. He is currently active in research in supercapacitor applications, power supply topologies, transient propagation and power conditioning area in power electronics. He has contributed over 130 papers to journals and international conference proceedings. He has authored and co-authored eight books and several of them are in power electronics. His latest book titled Energy Storage Devices for Electronic Systems: Rechargeable Batteries and Supercapacitors was published in 2015 by Elsevier AP, USA. Multiple patents were granted for his supercapacitor assisted circuit topologies after 2011. Prior to migrating to New Zealand in 2002, Kularatna was the CEO of the Arthur C Clarke Institute for Modern Technologies in Sri Lanka. 